\title{
Aeroacoustics of Three-Stream Jets
}

\author{
Brenda Henderson* \\ NASA Glenn Research Center, Cleveland, $\mathrm{OH}$
}

Results from acoustic measurements of noise radiated from a heated, three-stream, co-annular exhaust system operated at subsonic conditions are presented. The experiments were conducted for a range of core, bypass, and tertiary stream temperatures and pressures. The nozzle system had a fan-to-core area ratio of 2.92 and a tertiary-to-core area ratio of 0.96 . The impact of introducing a third stream on the radiated noise for third-stream velocities below that of the bypass stream was to reduce high frequency noise levels at broadside and peak jetnoise angles. Mid-frequency noise radiation at aft observation angles was impacted by the conditions of the third stream. The core velocity had the greatest impact on peak noise levels and the bypass-to-core mass flow ratio had a slight impact on levels in the peak jet-noise direction. The third-stream jet conditions had no impact on peak noise levels. Introduction of a third jet stream in the presence of a simulated forward-flight stream limits the impact of the third stream on radiated noise. For equivalent ideal thrust conditions, two-stream and threestream jets can produce similar acoustic spectra although high-frequency noise levels tend to be lower for the three-stream jet. 


\section{Fundamental Aeronautics Program}

Supersonics Project

\section{Aeroacoustics of Three-Stream Jets}

Brenda Henderson, NASA Glenn Research Center

18 ${ }^{\text {th }}$ AIAA/CEAS Aeroacoustics Conference June $4=6,2012$

Colorado Springs, Colorado www-nasa-gov
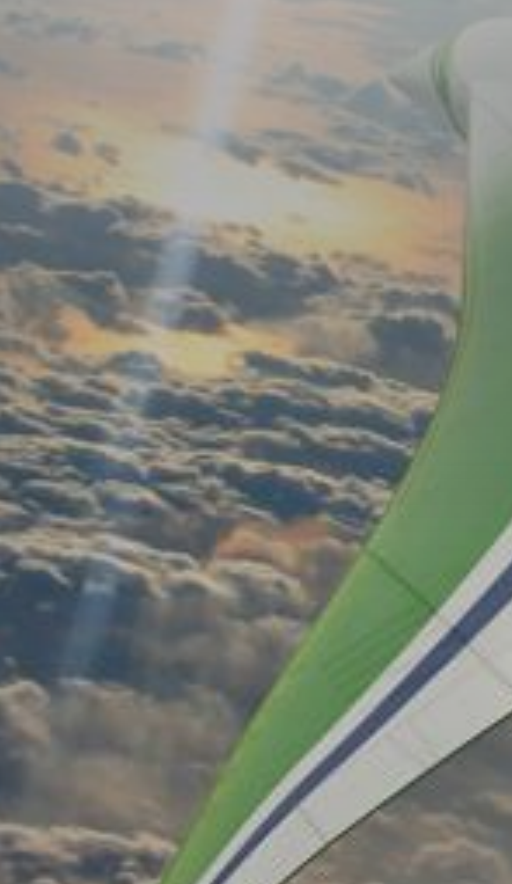

$\frac{5}{40}$
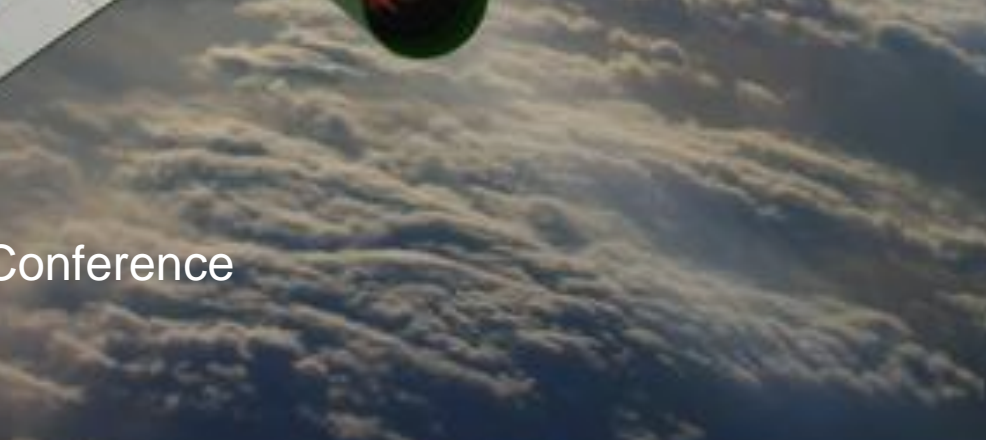


\section{Purpose of Study}

- Understand noise reduction potential of a third stream that may be available in future engine architectures

- Most straight-forward use of third stream is as an additional bypass stream

- Need to predict noise from three-stream jets - current semiempirical tools address single and dual stream jets

- Results of co-annular studies may guide other three-stream concepts (ejector) 


\section{Aero-Acoustic Propulsion Laboratory (AAPL)}

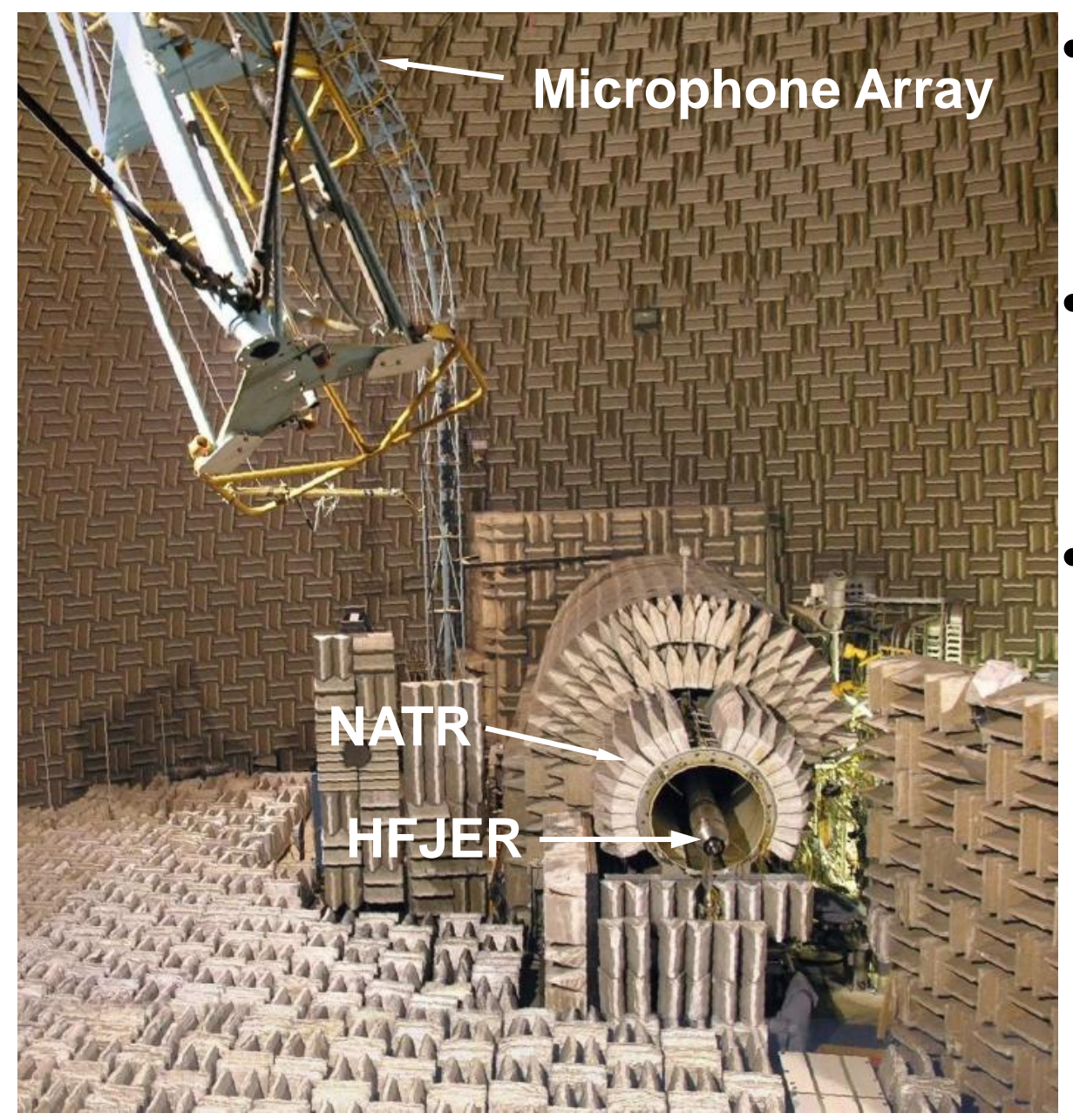

- AAPL

- 66 foot geodesic dome

- 45 foot microphone arc - 24 elements

- Nozzle Acoustic Test Rig (NATR)

- 53 inch simulated flight stream

- Maximum Mach number $=0.35$

- High Flow Jet Exit Rig (HFJER)

- 3-stream capability (3 ${ }^{\text {rd }}$ stream new)

- Independent pressure control on all streams

- Independent temperature control on fan and core streams

- Fan and third-stream temperatures the same 


\section{Model Hardware}

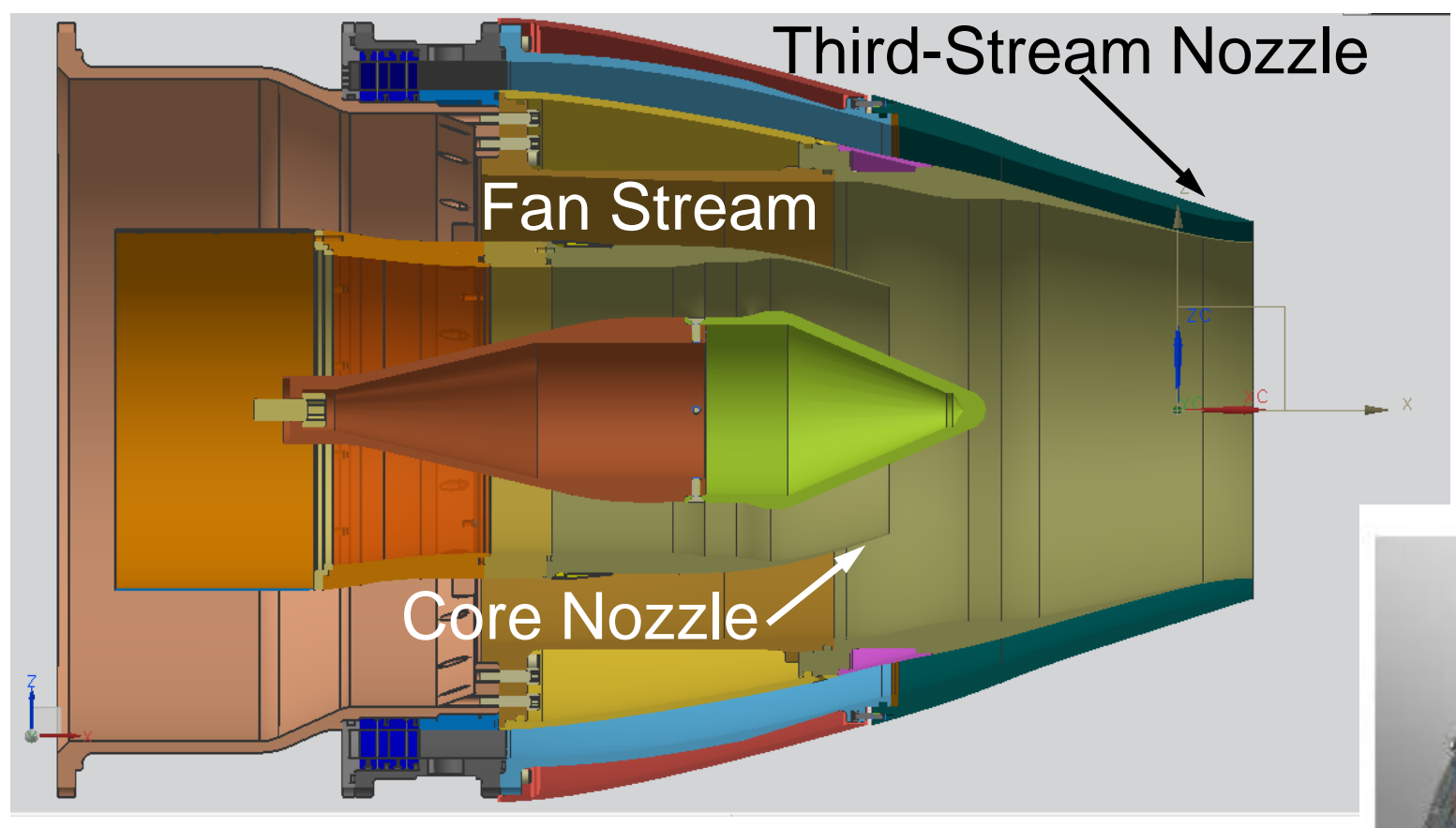

- Core stream nozzles

- Round

- Lobed mixer

- 4.8 inch exit diameter

- Fan-to-core area ratio $=2.92$ (fixed)

- Tertiary-to-core area ratio $=0.92$ (fixed) 


\section{Cycle Points}

\begin{tabular}{|c|c|c|c|c|c|c|}
\hline $\mathbf{N P R}_{\mathbf{c}}$ & $\mathbf{N P R}_{\mathbf{b}}$ & $\mathbf{N P R}_{\mathbf{t}}$ & $\mathbf{N T R}_{\mathbf{c}}$ & $\mathbf{M}_{\mathbf{f j}}=\mathbf{0}$ & $\mathbf{M}_{\mathbf{f j}}=\mathbf{0 . 2}$ & $\mathbf{M}_{\mathbf{f j}}=\mathbf{0 . 3}$ \\
\hline 1.5 & 1.5 & $1.0-1.5$ & 2.8 & $\checkmark$ & & \\
1.6 & 1.6 & $1.0-1.6$ & 2.8 & $\checkmark$ & & \\
1.7 & 1.7 & $1.0-1.7$ & 2.8 & $\checkmark$ & & \\
1.8 & 1.8 & $1.0-1.8$ & 2.8 & $\checkmark$ & & \\
\hline 1.5 & 1.5 & $1.0-1.5$ & 3.2 & $\checkmark$ & & \\
1.5 & 1.4 & $1.0-1.4$ & 3.2 & $\checkmark$ & & \\
1.5 & 1.6 & $1.0-1.6$ & 3.2 & $\checkmark$ & & \\
1.6 & 1.5 & $1.0-1.5$ & 3.2 & $\checkmark$ & & $\checkmark$ \\
1.6 & 1.6 & $1.0-1.6$ & 3.2 & $\checkmark$ & & $\checkmark$ \\
1.6 & 1.7 & $1.0-1.7$ & 3.2 & $\checkmark$ & & $\checkmark$ \\
1.7 & 1.6 & $1.0-1.6$ & 3.2 & $\checkmark$ & $\checkmark$ & $\checkmark$ \\
1.7 & 1.7 & $1.0-1.7$ & 3.2 & $\checkmark$ & $\checkmark$ & $\checkmark$ \\
1.7 & 1.8 & $1.0-1.8$ & 3.2 & $\checkmark$ & $\checkmark$ & $\checkmark$ \\
1.8 & 1.7 & $1.0-1.7$ & 3.2 & $\checkmark$ & & $\checkmark$ \\
\hline 1.8 & 1.8 & $1.0-1.8$ & 3.2 & $\checkmark$ & $\checkmark$ & $\checkmark$ \\
\hline
\end{tabular}

$\mathrm{NTR}_{\mathrm{b}}=1.25$

- $\mathrm{M}_{\mathrm{fj}}$ - free jet (simulated flight stream) Mach number

- NPR - nozzle pressure ratio

- NTR - nozzle temperature ratio

Subsonic Exhausts 


\section{Data Analysis}

- Baseline Experiments

$-\mathrm{NPR}_{\mathrm{c}}=\mathrm{NPR}_{\mathrm{f}}=1.8,1 \leq \mathrm{NPR}_{\mathrm{t}} \leq 1.8$

$-\mathrm{NTR}_{\mathrm{c}}=3.2$

$-\mathrm{M}_{\mathrm{fj}}=0$

- Reduced velocity of all streams

- Changed velocity ratio $\left(\mathrm{V}_{\mathrm{b}} / \mathrm{V}_{\mathrm{c}}\right)$

- Impact of velocity and bypass ratio

- Impact of simulated flight stream

- Impact of partially mixed flow

- Comparison on equal thrust basis 


\section{Repeat Acquisitions}
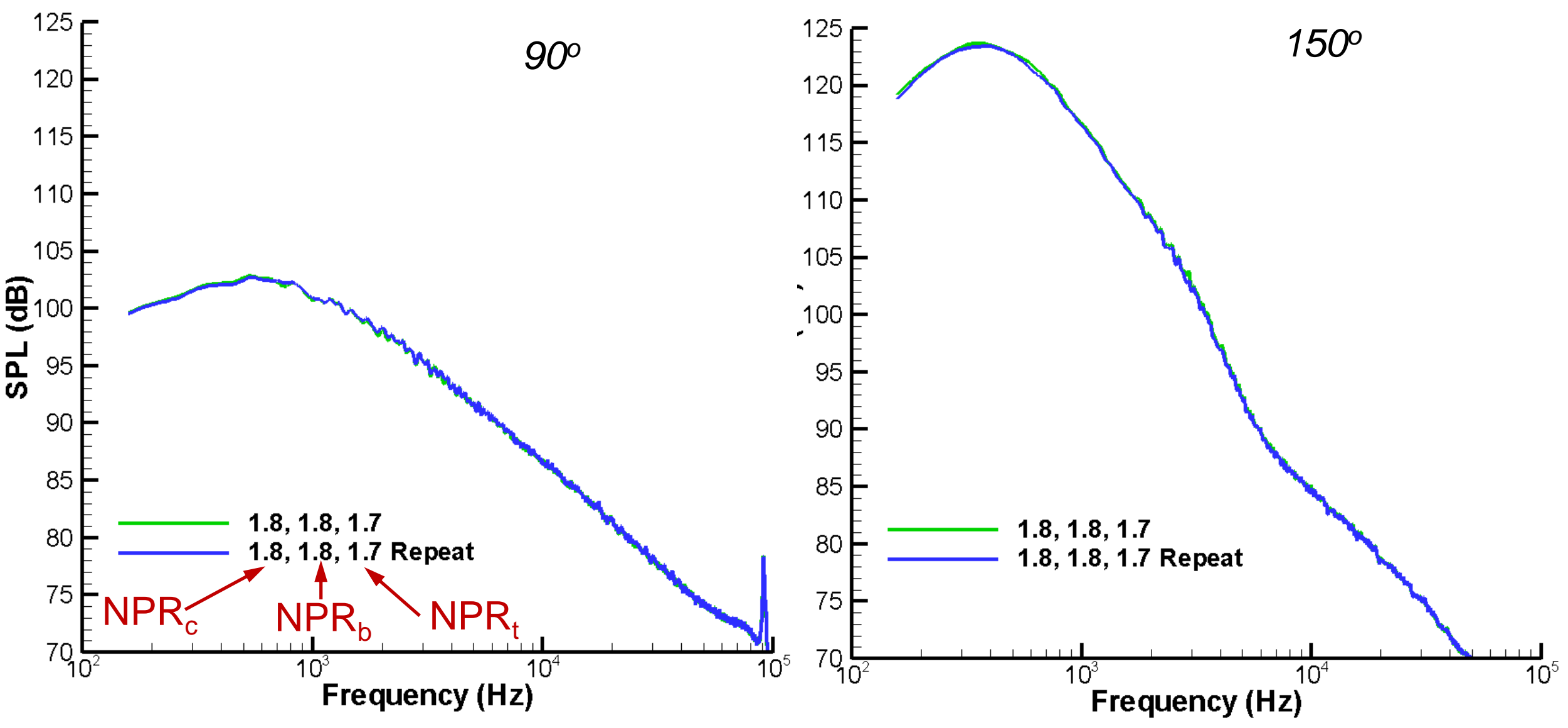


\section{Smoothed Data}
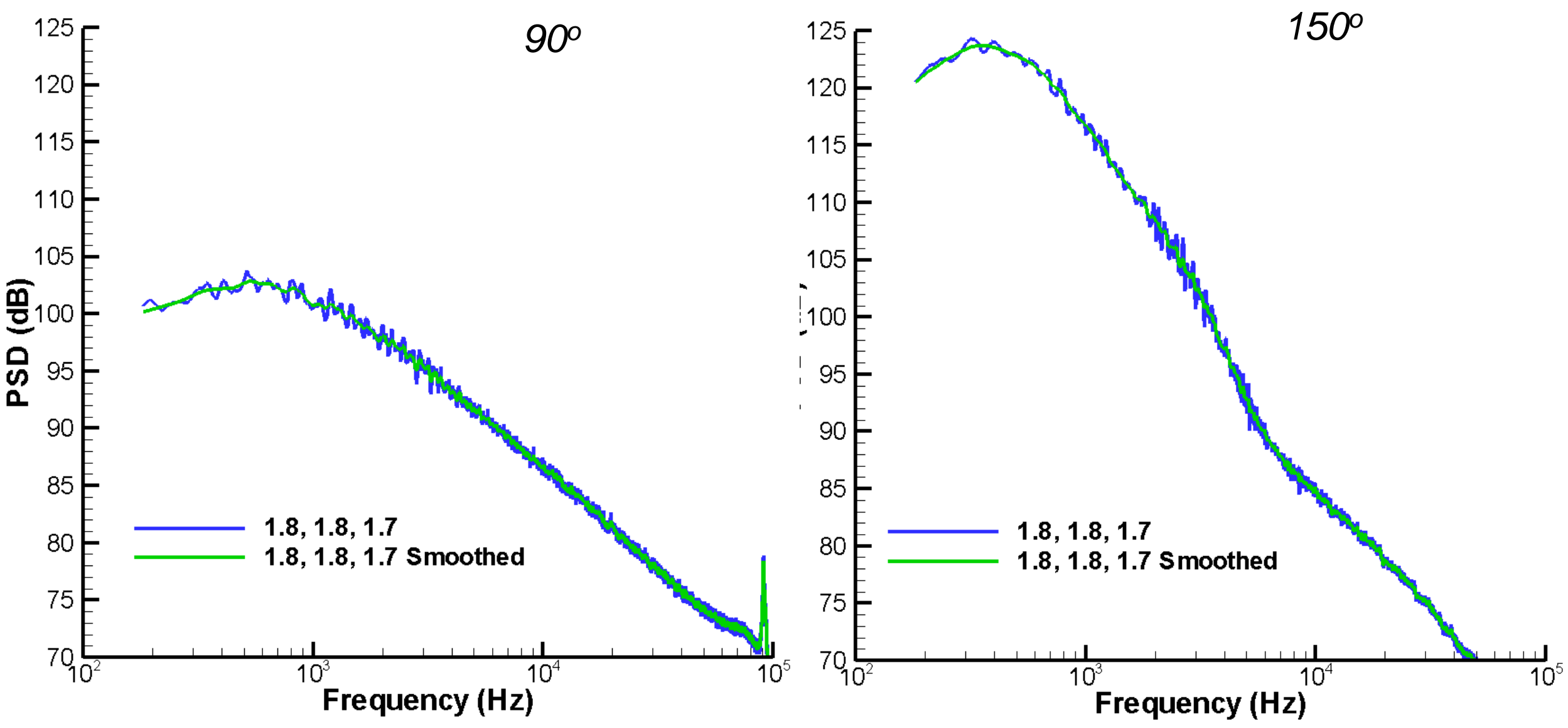


\section{Baseline Results}

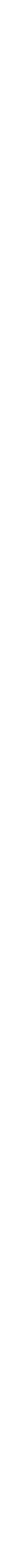




\section{Reduced Velocity Ratio $\left(V_{b} / V_{c}\right)$}
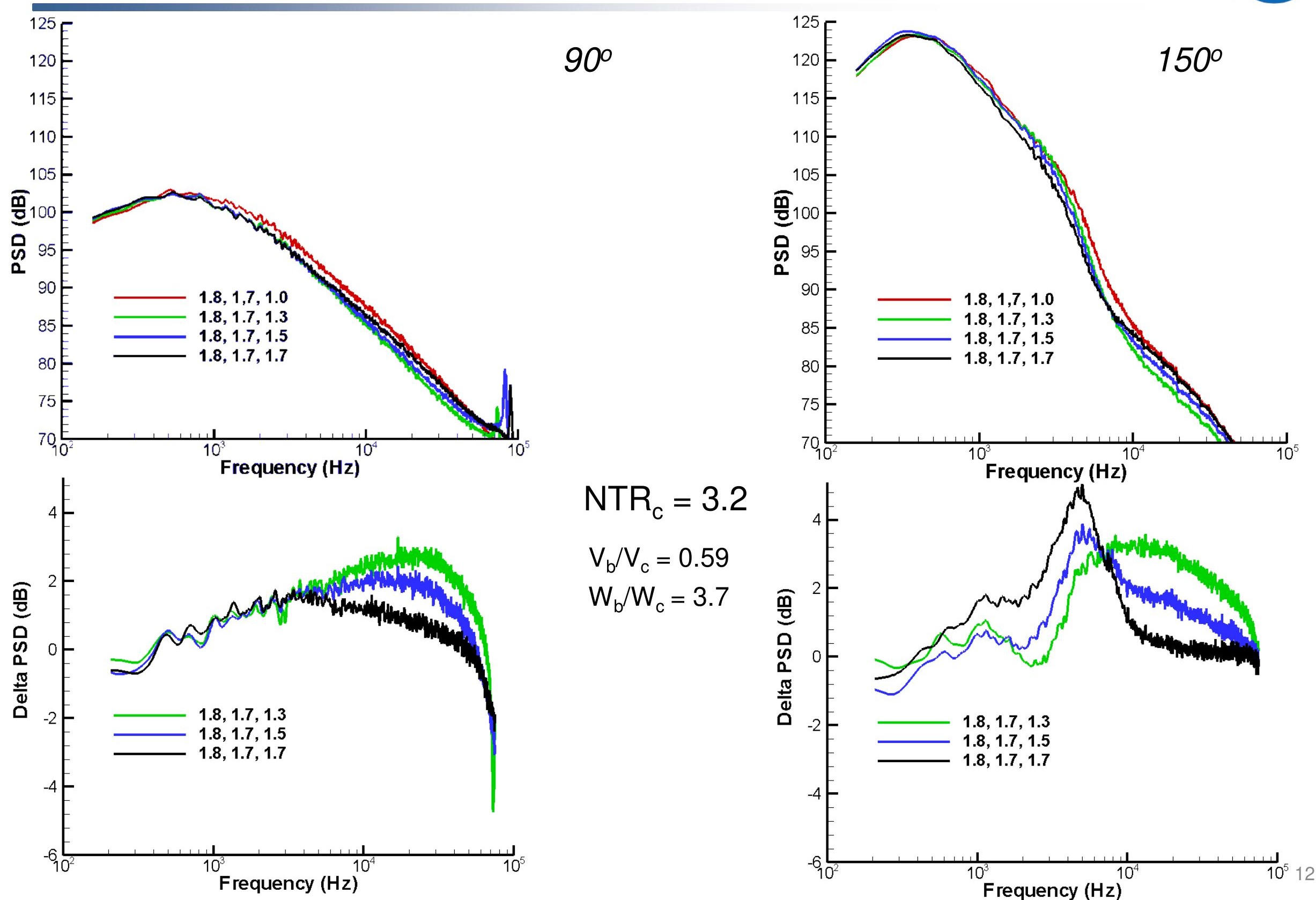


\section{Impact of Velocity and Bypass Ratio}
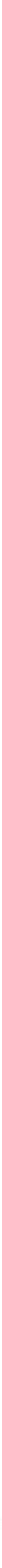


\section{Impact of Simulated Forward Flight}
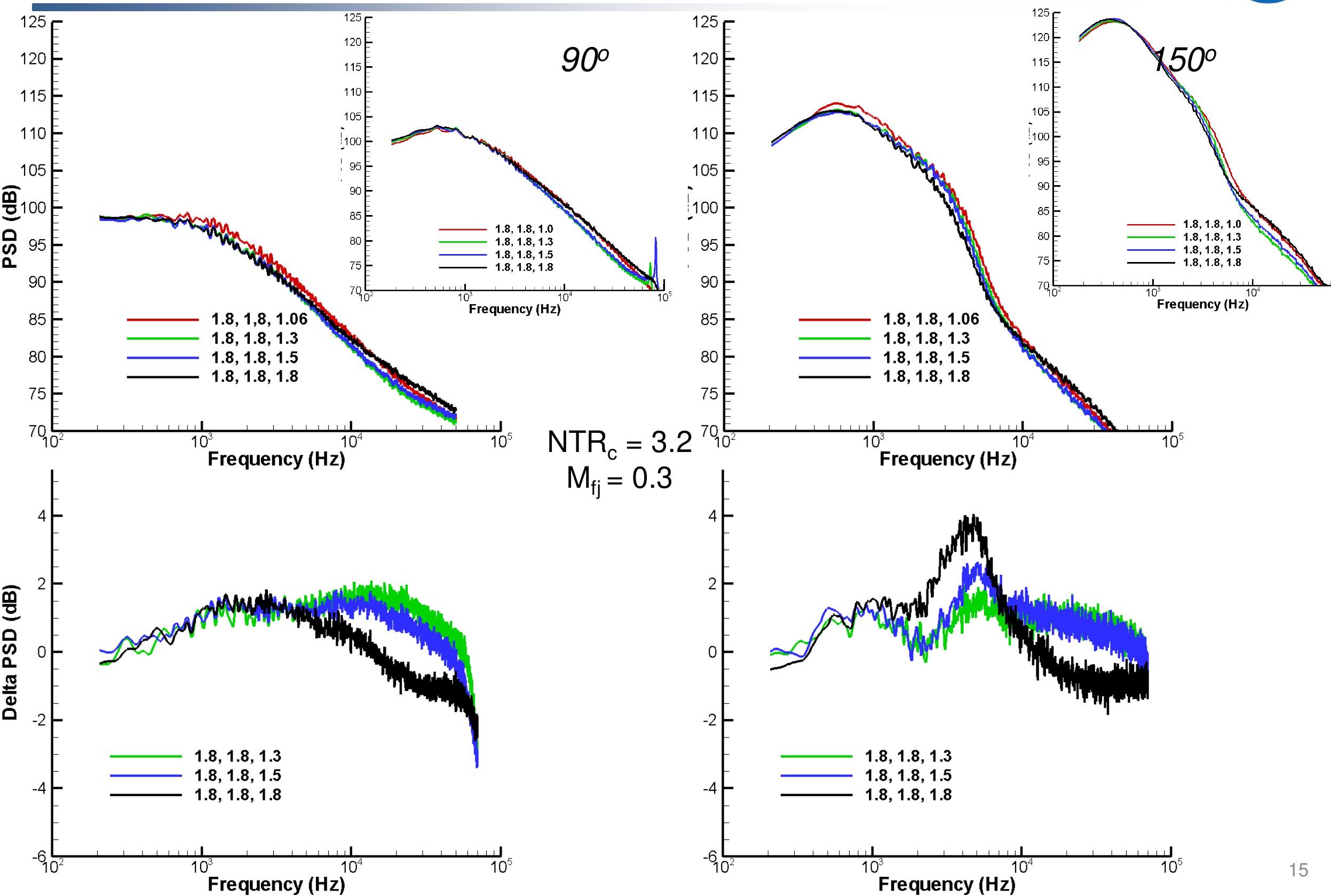


\section{Equal Thrust Comparisons}
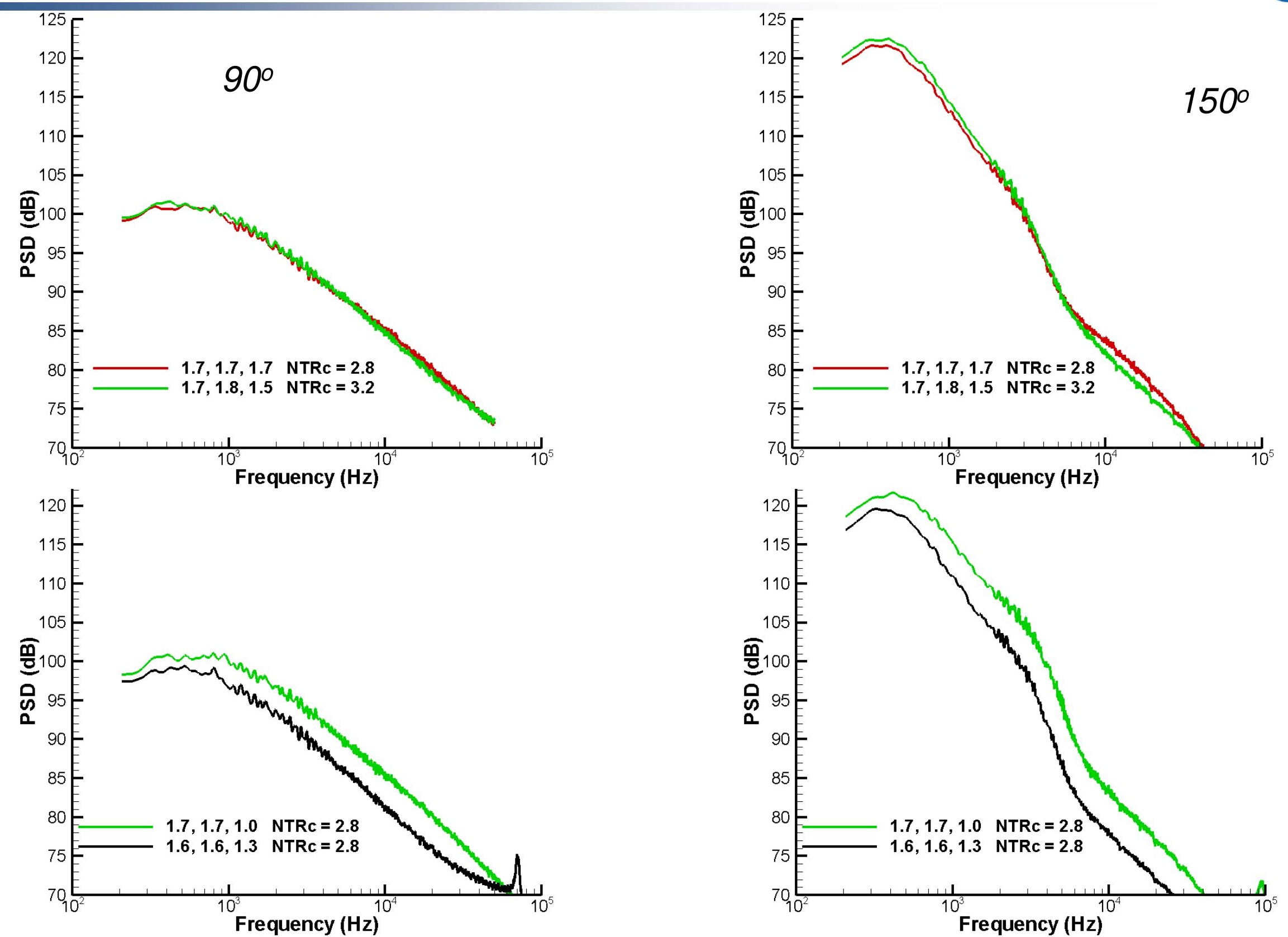


\section{Conclusions}

- Addition of a third stream reduced high-frequency noise and impacted mid-frequency noise

- The presence of a simulated flight stream reduced the impact of the third stream

- The core-stream velocity had the greatest impact of all parameters investigated on the radiated noise

- Comparisons on an equal thrust basis show that threestream jets are not inherently quieter than two-stream jets 